\title{
Conflicting pathology reports: a diagnostic dilemma
}

\author{
${ }^{*}$ Tal Shahar, MD, ${ }^{1}$ Uri Rozovski, MD, MHA, ${ }^{2}$ Yuval Shapira, MD, ${ }^{1}$ Erez Nossek, MD, ${ }^{1}$ \\ Bracha Zelikovich, BSc, ${ }^{5}$ Joseph Jossiphov, MD, ${ }^{3}$ Zvi Ram, MD, ${ }^{1}$ Andrew A. Kanner, MD, ${ }^{1}$ \\ Tali Siegal, MD, ${ }^{5}$ Deborah T. Blumenthal, MD, ${ }^{4}$ and Iris Lavon, $\mathrm{PhD}^{5}$
}

\begin{abstract}
Departments of ${ }^{1}$ Neurosurgery, ${ }^{2}$ Hematology and Bone Marrow Transplantation, ${ }^{3}$ Pathology, and ${ }^{4}$ Neuro-Oncology Service, Oncology Division, Tel Aviv Medical Center, Tel Aviv University School of Medicine, Tel Aviv; and 5Molecular Neuro-Oncology Laboratory, Gaffin Center for Neuro-Oncology, Department of Neurology, Hadassah Hebrew University Medical Center, Jerusalem, Israel
\end{abstract}

\begin{abstract}
The differential diagnosis of a brain lesion with two discordant pathology reports includes the presence of collision tumor, metaplastic changes, and labeling errors that occurred during the processing of the specimen. The authors present a case in which the first brain biopsy from a 47-year-old patient with a history of heavy smoking was compatible with metastatic small cell carcinoma, and the second biopsy taken during decompression craniotomy 3 weeks later was compatible with WHO Grade IV glioblastoma. Using short tandem repeat (STR) analysis of the two specimens and nontumorderived patient DNA, the authors found that the two specimens did not belong to the same individual. The authors conclude that allele imbalance or loss of heterozygosity detected by STR analysis is a reliable and valuable diagnostic tool for clarifying discrepancies in discordant pathology reports.
\end{abstract}

http://thejns.org/doi/abs/10.3171/2014.10.JNS131518

KEY WORDS glioblastoma; brain metastasis; clonality; collision tumor; loss of heterozygosity; LOH; diagnostic pathology errors; oncology

$\mathrm{S}$ HORT tandem repeat (STR) analysis is a polymerase chain reaction (PCR)-based technology that is based on typing highly polymorphic repeats of $2-6$ base pairs of DNA. Typically these repeats are inherited in codominant mode, so that the number of repeats in each allele is independently determined. Such analysis enables the identification of loss of heterozygosity $(\mathrm{LOH})$ or a shift in a genetic region, and reveals whether two cell populations are genetically "similar" or different. ${ }^{2}$ Emerging first as a DNA identity tool in the forensic setting, several clinical diagnostic uses for STR typing have already been established: for example, follow-up of engraftment after bone marrow transplantation; identification of uniparental disomy pattern of inheritance; and assessment of maternal cell contamination in prenatal specimens. ${ }^{13}$ The authors report a case in which STR typing was used to resolve a diagnostic dilemma involving two discrepant specimens from a patient with a brain tumor.

\section{Case Report}

History and Examination

A 47-year-old, right-handed man presented to the emergency center with a 2-day history of progressive right leg weakness. His medical history was significant for heavy smoking of 40 pack-years. His physical examination findings were remarkable for right lower-extremity weakness and mild upper-limb dysmetria. However, findings on chest radiographs were unremarkable, and brain MRI studies obtained with contrast revealed two separate ring-enhancing lesions localized to the left frontal lobe (Fig. 1). The radiological differential diagnosis included multiple brain metastases, multicentric glioblastoma, lymphoma, or, less likely, brain abscesses. A stereotactic biopsy sample taken from the left frontal anterior lesion was most compatible with metastatic small cell carcinoma with neuroendocrine features (Fig. 2A and B). At that time the working diagnosis, based on the history of heavy smoking, multiple brain lesions on MRI studies, and the pathology report was that of metastatic small cell lung carcinoma. Ten days after his initial presentation, the patient presented to the emergency center complaining of worsening right leg weakness, headaches, and blurred vision, and was admitted for urgent radiation treatment.

\section{Radiation Treatment and Subsequent Operation}

A chest CT scan performed at the time of admission

ABBREVIATIONS LOH = loss of heterozygosity; PCR = polymerase chain reaction; STR = short tandem repeat. ACCOMPANYING EDITORIAL See pp 273-275. DOI: 10.3171/2014.3.JNS14459.

SUBMITTED July 14, 2013. ACCEPTED October 22, 2014.

INCLUDE WHEN CITING Published online November 28, 2014; DOI: 10.3171/2014.10.JNS131518.

DISCLOSURE The authors report no conflict of interest concerning the materials or methods used in this study or the findings specified in this paper.

* Drs. Shahar, Rozovski, Blumenthal, and Lavon contributed equally to this report. 

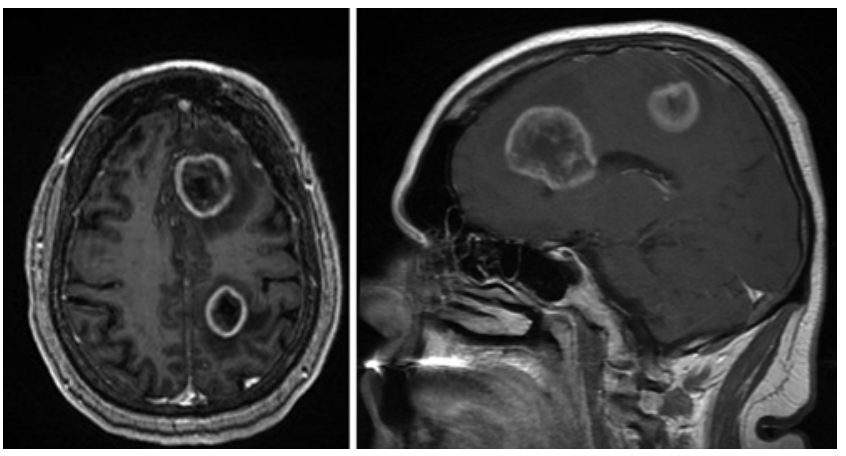

FIG. 1. Brain MRI studies obtained on admission. Axial (left) and sagittal (right) views demonstrating two separate ring-enhancing lesions localized to the left frontal lobe and associated with surrounding edema.

did not demonstrate any lung disease. While still undergoing whole-brain radiation, the patient's condition deteriorated. He became lethargic and complained of persistent headache associated with vomiting and progressive right hemiparesis. Brain CT scans demonstrated worsening mass effect with a midline shift due to increased peritumoral vasogenic edema. The patient underwent an urgent left frontal decompressive craniotomy and resection of the left frontal anterior lesion. Surprisingly, results of the histopathological examination of the resected tumor were most compatible with a primary CNS WHO Grade IV glial tu- mor, a glioblastoma (Fig. 2C and D). Of note, no evidence for metastatic carcinoma was found in this specimen.

\section{Histopathological Findings}

The differential diagnosis when two pathology reports on a brain tumor specimen obtained in a single patient do not concur includes the presence of a "collision tumor." This is a rare condition in which two histologically distinct neoplasms coexist within the same mass. The coexistence of glioblastoma with benign tumors has been sporadically described, as in the case of collision with epidermoid tumor, ${ }^{12}$ or with benign meningioma. ${ }^{14}$ Collision of glioma, and specifically glioblastoma, with metastatic carcinoma is extremely rare, and few well-documented cases are described. ${ }^{9}$ We considered that the interpretation of the tissue in the first pathology report as metastatic carcinoma may have in fact reflected sampling of glioblastoma tumor that underwent epithelial differentiation. Metaplastic changes of glioblastoma can result in tumor regions originating from the primary glioma clone, but are characterized by morphological and immunohistochemical features of carcinoma. ${ }^{4,9,11}$ In a number of cases in which metaplastic changes were reported, the initial diagnosis had been metastasis. ${ }^{10,11}$

To determine whether the two specimens in our case represented tumor originating from a single clone, we screened the DNA sequence of the two specimens and the white blood cells of the patient, representing the
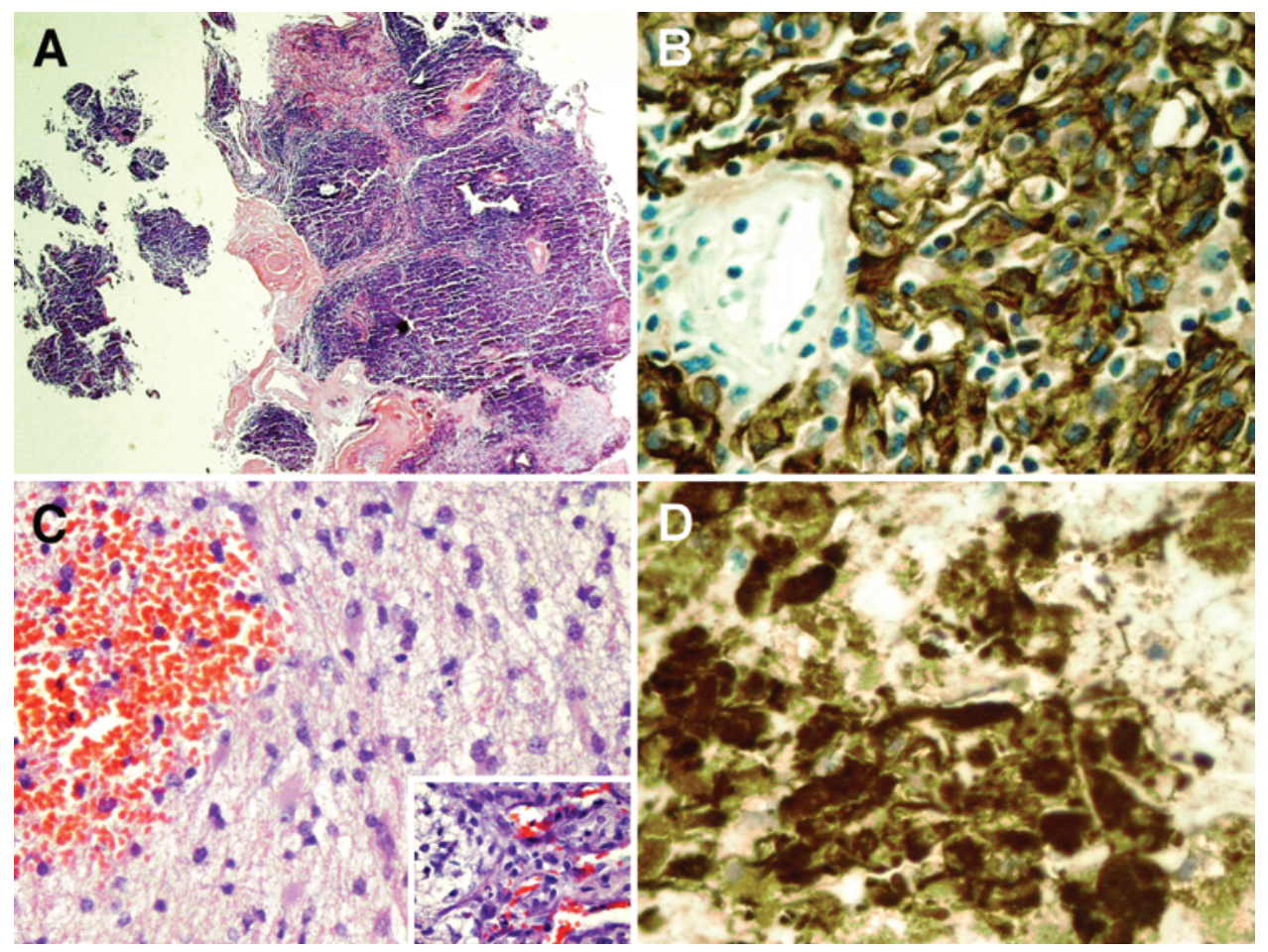

FIG. 2. Photomicrographs showing tissue sections used in histopathological studies. A and B: A stereotactically guided biopsy sample obtained in the left frontal anterior lesion revealed small cells with scant cytoplasm (A). The tumor cells stained positively for pankeratin (B) and weakly for synaptophysin (not shown), results that are most compatible with metastatic small cell carcinoma with neuroendocrine features. $C$ and $D$ : The resected specimen showed atypical astrocytes in a fibrillary background (C) that were positive for glial fibrillary acidic protein (GFAP) immunostaining (D). It also demonstrated areas of vascular proliferation (inset in C) and necrosis characteristic of WHO Grade IV glioblastoma. H \& E (A and C), pankeratin (B), and GFAP (D); original magnification $\times 5(A), \times 20(B), \times 10(C)$, and $\times 40(D$, inset in panel C). Figure is available in color online only. 
nontumor-derived DNA, in loci where genetic alterations are expected in glioblastoma. Loss of heterozygosity on chromosomal area $10 \mathrm{q}$, in the region of the $10 \mathrm{q} 23$ locus of the PTEN gene, is the most common genetic alteration, found in $60 \%-85 \%$ of patients. ${ }^{7}$ Loss of tumor suppressor genes located at the 1p, 14q, and 19q chromosomal areas has also been described. ${ }^{3}$ We therefore examined the two specimens for $\mathrm{LOH}$ in multiple genomic regions, namely, $10 \mathrm{q}, 1 \mathrm{p}, 14 \mathrm{q}$, and 19q. If the two specimens were derived from the same clone as in the case of epithelial differentiation, we expected to find the same $\mathrm{LOH}$ pattern in most or all loci. We also analyzed nontumor-derived DNA taken from the patient's white blood cells. This allowed us to distinguish between a single deletion of one allele and homozygosity, and to ensure that one or both specimens were in fact derived from the same patient (and not a laboratory error). As an example, the results of microsatellite analysis in two polymorphic loci at the 10q region are presented in Fig. 3, demonstrating that the tissue labeled "biopsy" and the tissue labeled "tumor resection" did not come from the same person. The same results were also obtained in the other alleles from the $1 \mathrm{p}, 14 \mathrm{q}$, and $19 \mathrm{q}$ regions.

Using this approach we unequivocally determined that the pathology report from the first biopsy and that from the resected tumor did not pertain to the same individual. The sample of the resected glioblastoma demonstrated $\mathrm{LOH}$ at several loci along the PTEN gene; however, the nondeleted alleles, equivalent in size, were present in the peripheral blood of the patient. The analysis of the first biopsy did not demonstrate the typical findings in a glioblastoma patient with $\mathrm{LOH}$ at chromosome 10, and the alleles at these loci were different in size compared with what was found in the patient's peripheral blood. We therefore concluded that the first pathology specimen belonged to a different individual (Fig. 3).

\section{Discussion}

A book titled To Err is Human: Building a Safer Health System, published in 1999, determined that at least 44,000 people die in hospitals in the US each year as a result of medical errors, and it focused public attention on this major public health issue..$^{5}$ In surgical pathology, labeling errors occur when the specimen is labeled with an incorrect patient name or identification number (a patient identification error). Labeling errors can also occur when the specimen is misidentified as to site of origin or time of collection, although correctly associated with patient name and/or unique identification number (specimen identification error). The error can further be classified as preanalytical if it occurs during the process of receiving and preparing the surgical specimen; analytical if due to misinterpretation by the pathologist; or postanalytical if it occurs during the process of conveying results to the clinician. ${ }^{6}$

Few studies have addressed the issue of surgical specimen labeling errors: in a review of 21,351 surgical specimens, $11(0.04 \%)$ involved specimens that were associated with an incorrect patient assignment. ${ }^{8}$ Layfield reviewed 29,479 consecutive cases over a period of 18 months to estimate the rate of errors that occur during the preanalyti-
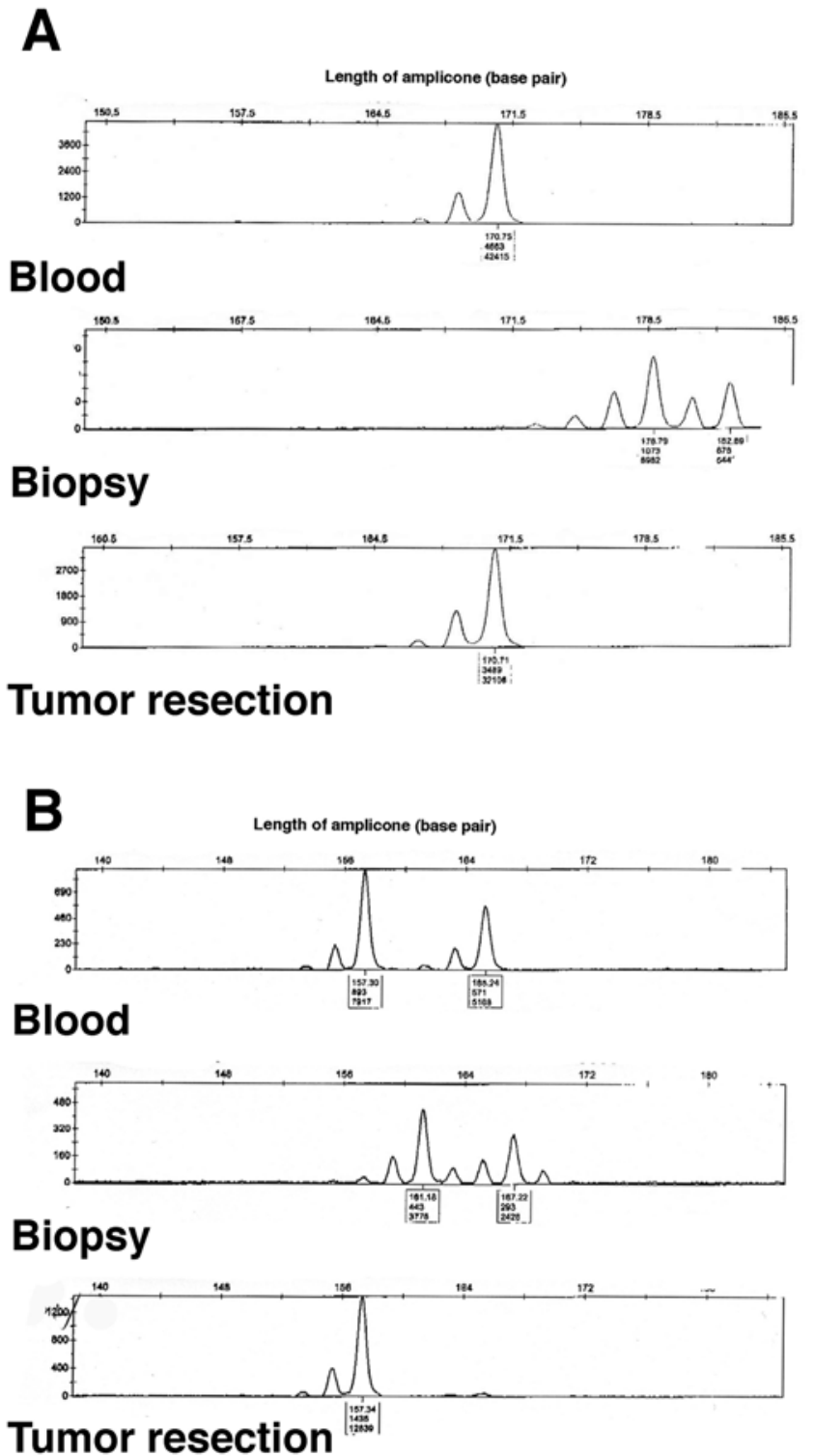

FIG. 3. Graphs showing results of a microsatellite analysis in two polymorphic loci at the $10 \mathrm{q}$ region in our patient. For each locus, PCR products are shown separately from nontumor-derived DNA (upper bars in both panels), tissue biopsy (center bars), and from the resected tumor (lower bars). The peak's height represents fluorescent intensity (which is an indication of the PCR product quantity), and the number below the peak indicates the size of the PCR product in the base pair. A: The upper bar demonstrates a single allele for the examined locus in the patient's blood specimen, which indicates that this patient is homozygous for that specific allele. This is also seen in the specimen taken from the resected tumor (lower bar). However, this is not the case in the biopsy specimen (center bar), which demonstrates two different alleles (sizes 178 and 182). This demonstrates that the blood and resected tumor specimens were taken from the same person, whereas the tissue of the biopsy was taken from a different person. B: The second polymorphic locus in the $10 \mathrm{q}$ region demonstrated two alleles in the blood specimen (sizes 157 and 165, upper bar) and two different alleles in the biopsy specimen (sizes 151 and 167, center bar). The tissue from the resected specimen demonstrates a single allele (size 157, lower bar), identical to one of the two alleles that were present in the blood specimen, suggesting an $\mathrm{LOH}$ in this region in the tumor. 
cal phase at the time of specimen processing. Seventy-five labeling errors $(0.25 \%)$ were found: $55(73 \%)$ involved the patient name; most occurred at the gross processing room and involved the mislabeling of cassettes, with subsequent blocks or slides being associated with an incorrect patient or an incorrect site. ${ }^{6}$ These numbers probably underestimate the true labeling error rate during the preanalytical phase. For example, in our case, only extensive genetic workup revealed that such an error had occurred during the preanalytical phase. To prevent repeat of similar errors in our facility, a root cause analysis was undertaken. A flow diagram of the preanalytical phase revealed a complex process with the potential for labeling errors along several points. Because the pathological description of the specimen at the time the surgical biopsy sample arrived in the pathology laboratory was compatible with a stereotactically guided needle biopsy taken from a brain tumor, we concluded that the correct specimen arrived and that the error occurred during the gross processing in the pathology laboratory. The investigation revealed that a tissue pickup error occurred, most probably when extraneous tissue was placed inadvertently in the cassette of the studied cases, and was therefore present in all slides and sections.

This investigation led to a thorough revision of the guidelines at the pathology laboratory's gross processing room. Based on the Standard Operation Procedure guidelines we now emphasize avoiding pickup errors. Procedures for maintaining meticulous cleanliness of the working environment, cleaning of the gross processing and embedding forceps between every case, cleaning of scalpels after every use, and the use of tissue bags for specimens with small fragments were revised and are now strictly enforced. Studies by the College of American Pathologist Q-Probes quality improvement program identified $1.5 \%$ of pathology cases with extraneous tissue $(1486 / 97,425)$ in a prospective study and $6.2 \%$ of pathology cases $(1078 / 17,422)$ in a retrospective study. In both studies, "floaters" (tissue that was picked up after microtomy and thus present in only one section of the slide) were the most common reason for the presence of extraneous tissue. Tissue pickup errors were less common, but were found in $28.4 \%$ and $15.9 \%$ of the prospective and retrospective extraneous cases, respectively. ${ }^{1}$ These findings and our case report highlight the fact that pathology errors do occur, and hence the need for vigilance from the entire chain of medical service providers, including the neurosurgeons.

Fortunately, in this case the error was identified early in the course of treatment and prompted a modification in the radiation plan and the addition of the alkylating agent temozolomide to the treatment regimen, as is consistent with standard of care for glioblastoma. In this report we highlight the occurrence of a diagnostic labeling error and suggest that in cases in which pathology reports conflict, or do not correlate with the clinical findings, allele imbalance or $\mathrm{LOH}$ fingerprinting can help in determining the correct diagnosis.

\section{References}

1. Gephardt GN, Zarbo RJ: Extraneous tissue in surgical pathol- ogy: a College of American Pathologists Q-Probes study of 275 laboratories. Arch Pathol Lab Med 120:1009-1014, 1996

2. Junker K, Thrum K, Schlichter A, Müller G, Hindermann W, Schubert J: Clonal origin of multifocal renal cell carcinoma as determined by microsatellite analysis. J Urol 168:26322636, 2002

3. Kaneshiro D, Kobayashi T, Chao ST, Suh J, Prayson RA: Chromosome 1p and 19q deletions in glioblastoma multiforme. Appl Immunohistochem Mol Morphol 17:512-516, 2009

4. Kepes JJ, Fulling KH, Garcia JH: The clinical significance of "adenoid" formations of neoplastic astrocytes, imitating metastatic carcinoma, in gliosarcomas. A review of five cases. Clin Neuropathol 1:139-150, 1982

5. Kohn LT, Corrigan JM, Donaldson MS: To Err Is Human: Building a Safer Health System. Washington, DC: National Academy Press, 2000

6. Layfield LJ, Anderson GM: Specimen labeling errors in surgical pathology: an 18-month experience. Am J Clin Pathol 134:466-470, 2010

7. Li J, Yen C, Liaw D, Podsypanina K, Bose S, Wang SI, et al: PTEN, a putative protein tyrosine phosphatase gene mutated in human brain, breast, and prostate cancer. Science 275:1943-1947, 1997

8. Makary MA, Epstein J, Pronovost PJ, Millman EA, Hartmann EC, Freischlag JA: Surgical specimen identification errors: a new measure of quality in surgical care. Surgery 141:450-455, 2007

9. Mörk SJ, Rubinstein LJ: Metastatic carcinoma to glioma: a report of three cases with a critical review of the literature. $\mathbf{J}$ Neurol Neurosurg Psychiatry 51:256-259, 1988

10. Mørk SJ, Rubinstein LJ, Kepes JJ, Perentes E, Uphoff DF: Patterns of epithelial metaplasia in malignant gliomas. II. Squamous differentiation of epithelial-like formations in gliosarcomas and glioblastomas. J Neuropathol Exp Neurol 47:101-118, 1988

11. Mueller W, Lass U, Herms J, Kuchelmeister K, Bergmann M, von Deimling A: Clonal analysis in glioblastoma with epithelial differentiation. Brain Pathol 11:39-43, 2001

12. Muzumdar DP, Goel A, Desai KI: Pontine glioma and cerebellopontine angle epidermoid tumour occurring as collision tumours. Br J Neurosurg 15:68-71, 2001

13. Pfeifer JD, Zehnbauer B, Payton J: The changing spectrum of DNA-based specimen provenance testing in surgical pathology. Am J Clin Pathol 135:132-138, 2011

14. Vaquero J, Coca S, Martínez R, Jiménez C: Convexity meningioma and glioblastoma in collision. Surg Neurol 33:139-141, 1990

\section{Author Contributions}

Conception and design: Shahar, Lavon. Acquisition of data: Shahar, Shapira, Zelikovich, Jossiphov, Kanner, Blumenthal, Lavon. Analysis and interpretation of data: Shahar, Zelikovich, Jossiphov, Kanner, Siegal, Blumenthal, Lavon. Drafting the article: Shahar, Rozovski. Critically revising the article: all authors. Reviewed submitted version of manuscript: all authors. Approved the final version of the manuscript on behalf of all authors: Ram. Administrative/technical/material support: Ram, Shahar, Shapira, Kanner, Siegal, Blumenthal, Lavon. Study supervision: Ram, Shahar.

\section{Correspondence}

Zvi Ram, Department of Neurosurgery, Tel Aviv Medical Center, 6 Weizman St., Tel Aviv 64239, Israel. email: zviram@tlvmc. gov.il. 